\title{
Valsalva Retinopathy in Pregnancy: A Case Report from the Emergency Department
}

\section{Edward C. O’Bryan ", Michael D. Green and Lacey P. MenkinSmith}

Department of Emergency Medicine, Medical University of South Carolina, Charleston, SC, USA

*Corresponding author: Edward C. O'Bryan, Department of Emergency Medicine, Medical University of South Carolina, Charleston, SC, USA, Tel: (843)-792-2123; Email: obryanec@musc.edu

Received date: January 4, 2018; Accepted date: January 15, 2018; Published date: January 20, 2018

Copyright: (c) 2017 O'Bryanc EC, et al. This is an open-access article distributed under the terms of the Creative Commons Attribution License, which permits unrestricted use, distribution, and reproduction in any medium, provided the original author and source are credited.

\begin{abstract}
Introduction: Rapid increases in intraocular pressure can result in sudden-onset vision loss in a self-limiting condition known as Valsalva Retinopathy. Pregnancy is a known risk factor.

Case Presentation: A pregnant woman presents to the Emergency Department with complaints of unilateral central vision loss after an episode of vomiting. Fundus exam of the left eye identified haemorrhages consistent with Valsalva Retinopathy.

Conclusion: Superficial retinal capillaries can hemorrhage as a result of Valsalva maneuvers. Natural pregnancy changes with other stressors like vomiting can leave a woman at higher risk for Valsalva retinopathy. Valsalva Retinopathy patients can be discharged with close follow-up.
\end{abstract}

\section{Introduction}

The Valsalva maneuver is a forceful expiration against a closed glottis that causes increased intra-thoracic or intra-abdominal pressures $[1,2]$ It is associated with events such as coughing, sneezing, vomiting, strain with stooling, weightlifting, and pushing during delivery $[1,4]$. These pressures can be transmitted through the valveless orbital vessels, rapidly increasing intraocular pressure and causing rupture of superficial retinal capillaries [3-5]. The haemorrhage that ensues can cause sudden-onset vision loss in a self-limiting, and welldescribed condition known as Valsalva Retinopathy (VR) [1,3-5]. Pregnancy, due to common physiologic mechanisms, is a known risk factor for VR [6-14]. We present a case of VR in a young woman in her 36 th week of pregnancy.

\section{Case Presentation}

A 19 -year old woman in her $36^{\text {th }}$ week of pregnancy presented to the Emergency Department from an outside hospital with complaints of unilateral central vision loss. The night before presentation, she had an episode of vomiting that was immediately followed by painless vision loss in the left eye. The central vision loss remained constant throughout the following day.

The patient was otherwise healthy and she denied any other vision changes. She denied wearing glasses or contact lenses, using steroids, history of trauma, autoimmune conditions, bleeding diatheses, vascular disease, or diabetes. The patient had not experienced similar episodes prior to this one. The patient had hyperemesis gravidarum earlier in her pregnancy. Her past medical history was only remarkable for post-concussion syndrome, goitre, smoking one half pack of cigarettes per day and a remote childhood history of strabismus that self-corrected.

On exam in the ED, the patient's blood pressure was transiently elevated to $151 / 87$ and then decreased to $134 / 64$ four and half hours later. Her pulse was first documented at 98 and after 4.5 hours, she was tachycardic to $102 \mathrm{bpm}$. Her vitals were otherwise unremarkable. The patient noted that in her left eye, central vision was lost but her peripheral vision was intact. There was neither visible foreign body nor fluorescein uptake.

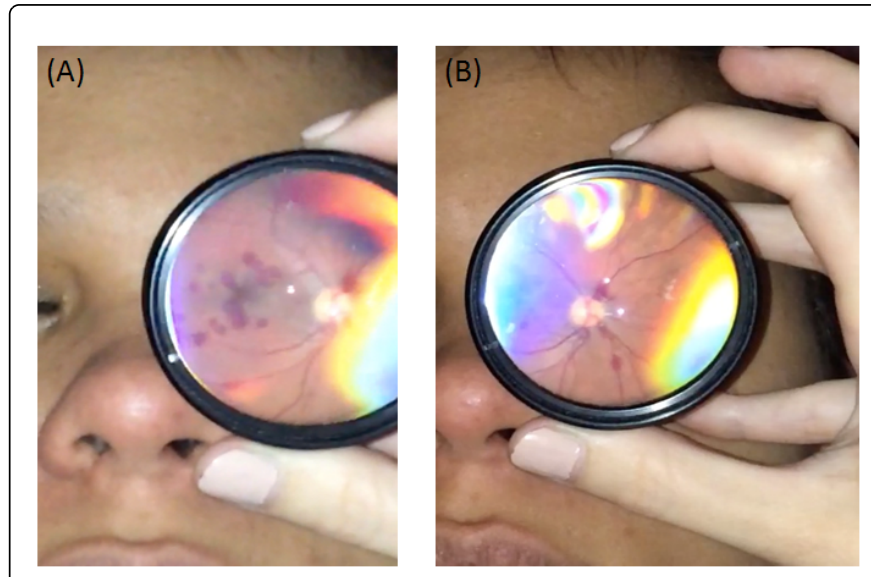

Figure 1: Pathognomonic hemorrhages of Valsalva Retinopathy on first day after vision loss. Fundus exam identified (A) perifoveal hemorrhages, (B) 7-10 intra-retinal hemorrhages without exudate or thickening in the macula, intra-retinal and sub-retinal hemorrhages outside of the macula in the posterior pole.

Ophthalmology was consulted, and their exam demonstrated 20/20 visual acuity in her right eye and around the central scotoma in her left eye. Tonometry was $13 \mathrm{mmHg}$ on the right and $14 \mathrm{mmHg}$ on the left. Pupillary exam, eye movement, and slit lamp evaluations were unremarkable. Fundus exam of the left eye identified a superonasal and inferonasal haemorrhages around a pink disc with sharp borders and a 
healthy rim, 7-10 intra-retinal haemorrhages without exudate or thickening in the macula (Figure 1), a few intra-retinal and sub-retinal haemorrhages outside of the macula in the posterior pole. VR is a clinical diagnosis based on the patient's features and history, VR was confirmed; labs were not necessary at this point. Conservative management (i.e. watch-and-wait, versus laser therapy) was recommended with follow-up in 2 weeks. The patient was counseled that this condition usually resolves without further intervention in 1-3 months, but she was also encouraged to return if symptoms changed or worsened.

\section{Discussion}

VR was first described by Duane in 1972, as an effect of rapid increases in backward venous pressures to the retinal capillaries resulting in a spectrum from mild retinal edema to, most severely, preretinal haemorrhages [3]. He associated the severity with the size of the compressive force from the Valsalva stimulus [3]. These haemorrhages prefer the macula, but may result in detachment of the internal limiting membrane causing characteristically sub-hyaloid, and less frequently sub-retinal, retinal, and vitreous haemorrhages [1,12-14]. This can happen bilaterally, but it is predominantly unilateral in presentation. Prognosis is usually good, with return to complete vision in 1 week to 10 months using conservative management with close observation $[1,8]$. Prognosis can be negatively affected by pigment changes in the macula representing photoreceptor toxicity. Toxicity can occur due to prolonged exposure to hemosiderin associated with protracted observation of larger haemorrhages. For the cases where this is a possibility or cases where the patient wishes for an expedited recovery, alternative treatments include the neodymium: yttrium aluminum garnet laser (Nd:Yag) membranotomy or vitrectomy. Complications with Nd:Yag include, albeit rarely within the first six months, persistent pre-macular cavity formation, retinal detachment, epiretinal membrane formation or a macular hole. Vitrectomy, while immediately effective in most cases, can be complicated by macular hole, cataract, and retinal artery microaneurysm $[7,12]$.

The etiology for sudden onset unilateral vision disturbance has a wide differential, including anaemia, coagulopathies, hyper-viscosity, leukaemia's, vascular occlusive disease, elevation changes, HTN and vascular occlusive disease, and idiopathic [16]. Valsalva maneuvers have also been reported as resulting in subconjunctival haemorrhages, periorbital skin petechiae, and exophthalmos from orbital haemorrhage, $[5,11]$ VR usually occurs in healthy adults in the context of strenuous activity including lifting, sexual intercourse, straining during constipation or labor, sneezing, coughing, or vomiting [6-11] It is appropriate to rule out possible systemic disease that may result in retinal haemorrhages $[5,16]$. Our patient was in her third trimester and she was referred from an outside hospital where she was getting routine care, so any residual sequelae could be followed up by her primary team. Our patient's history and clinical features clearly demonstrated a classic presentation of VR.

\section{VR and Pregnancy}

The pregnant state includes multiple anatomic, hormonal, hematologic, metabolic, immunologic changes, making it a risk factor for VR. Physiologic thrombocytopenia during pregnancy can increase potential for haemorrhage. Increased uterine size during pregnancy can elevate intra-abdominal pressures [9]. The additional and sudden increases in intra-abdominal pressures of vomiting as in Hyperemesis Gravidarum, can be enough compressive force to rupture superficial retinal capillaries [7]. Alternatively, spontaneous vaginal delivery can be associated with enough sustained pressures against a closed glottis to rupture retinal capillaries. Enah presents a case of VR with only mild visual disturbance, that occurs one day after vaginal delivery [6]. While labor can be a theoretical risk factor for exacerbation or cause of pre-retinal haemorrhage, so can general and epidural anesthesias [2]. The hypoxia and hypercarbia associated with general anaesthesia can result in vasodilation and concomitant increases in retinal venous pressures; the additional increase in intra-thoracic pressures from extubation can cause enough of an increase in retinal capillaries to result in a VR $[2,6,7,11,12]$. Epidural anaesthesia can cause an increase in Cerebral spinal fluid pressure secondary to mass effect in the epidural space also resulting in VR [6,7]. Accordingly, there is no reason to opt for cesarean section as an alternative to spontaneous vaginal delivery, as it is not yet known to positively affect prognosis of VR, and it contains risks of exacerbation itself. Options, risks, and benefits should be discussed between the ophthalmologist and the obstetrician $[8,12]$. Ultimately, it is best practice to present all risks to the patient and let her input guide the final decision [7].

Perhaps in pregnant patients, psychiatric history should be observed closely by all professionals involved, as there have been documented cases of post-partum depression attributed to the conservative management of VR. Postpartum depression can be linked with decreased interest and consequently decreased care of infants [16].

\section{Conclusion}

The case presented demonstrates the classic features of VR. VR is a self-limiting condition with a good prognosis, and it can be managed conservatively by the Emergency Department, when diagnosis is confirmed by ophthalmic exam. Pregnancy is a known risk factor, however there is no evidence supporting alternative antenatal course, nor alternative mode of delivery. Long-term management includes a team approach with clear follow up instructions by the Emergency team, and clear communication between obstetrician, ophthalmologist, and patient.

\section{References}

1. Chapman-Davies A, Lazarevic A (2002) Valsalva maculopathy. Clin Exp Optom 85: 42-45.

2. Hönemann C, Brandt L (2015) Valsalva Retinopathy: A Rare Complication After General Anesthesia. Anes Anal Cas Rep 5: 231-233.

3. Duane TD (1973) Valsalva Hemorrhagic Retinopathy. T Am Ophthal Soc 70: 298-313.

4. Callender D, Beirouty ZAY, Saba SN (1995) Valsalva Hemorrhagic Retinopathy in a Pregnant Woman. Eye 9: 808-809.

5. Geyer O, Wasserman D, Rothkoff L, Lazar M (1990) Orbital Haemorrage induced by labor. Br J Ophthalmol 74: 242.

6. Enah A, Almeida D (2013) Valsalva hemorrhagic retinopathy during labor: a case report and literature review. Can J Ophthalmology 48: $145-147$.

7. Wickremasinghe SS, Tranos PG, Davey C (2003) Valsalva haemorrhagic Retinopathy in a pregnant woman: implications for delivery. Acta Ophthalmologica Scandinavica 81: 420-422.

8. Ramskold LAH, Asaria RH (2012) Valsalva Retinopathy secondary to hyperemesis gravidarum. Eur J Obstet Gynecol Reprod Biol 162: 118-119.

9. Tara F, Sharifi M, Hoseini E (2015) Valsalva Retinopathy in pregnancy: a case report. BMC Res Notes 8: 67.

10. Al-Mujaini AS, Montana CC (2008) Valsalva Retinopathy in pregnancy: a case report. J Med Case Reports 2: 101. 
Citation: O'Bryanc EC, Green MD, MenkinSmith LP (2018) Valsalva Retinopathy in Pregnancy: A Case Report from the Emergency Department. J Eye Dis Disord 3: 114.

Page 3 of 3

11. Ladjimi A, Zaouali S, Messaoud R, Ben Yahia S, Attia S, et al. (2002) Valsalva Retinopathy Induced by labour. Eur J Ophthalmol 12: 336-338.

12. El-Defrawy H, Sallam A, McKechnie CJ, Zambarakji H (2011) Valsalva Retinopathy in pregnancy: should we treat? JRSM Short Rep 2: 45.

13. Liu Z, Pan X, Bi H (2014) Treatment of Valsalva Retinopathy. Optom Vis Sci 91: 278-281.

14. Daglioglu MC, Coskun M (2013) Posterior Hyaloidotomy by Nd:YAG Laser Application in a Patient with Postpartum Depression Caused by Valsalva Retinopathy. Cas Rep Ophthalmol 4: 64-68.
15. Mathew DJ, Sarma SK (2016) Valsalva retinopathy with double ring sign: Laser membranotomy for twin bleeds. Saudi J Ophthalmol 30: 6-7.

16. Graverson VAK, Jampol LM, Meredith T, Landers M, Slakter J, et al. (2014) Hemorrhagic Unilateral Retinopathy. Retina 34: 483-489. 